\title{
Positive Predictive Value of Abdominal Sonography in the Diagnosis of Ischemic Colitis
}

Authors

Affiliations

\section{E. López ${ }^{1}$, T. Ripolles ${ }^{1}$, M. J. Martinez ${ }^{1}$, P. Bartumeus ${ }^{2}$, J. Blay ${ }^{1}$, A. López ${ }^{3}$}

${ }^{1}$ Radiology, Hospital Universitario Doctor Peset, Valencia, Spain

${ }^{2}$ Radiology, Hospital Universtiario San Juan, San Juan, Spain

${ }^{3}$ Gastroenterology, Hospital Universitario Dr Peset, Valencia, Spain
Key words

ultrasound

ischemia/infarction

- abdomen received $\quad 17.04 .2015$

accepted 27.07.2015

Bibliography

DOI http://dx.doi.org/

10.1055/s-0035-1559775

Published online:

November 6, 2015

Ultrasound International Open

2015; 1: E41-E45

(c) Georg Thieme Verlag KG

Stuttgart · New York

ISSN 2199-7152

Correspondence

\section{Dr. Tomas Ripolles}

Radiology

Hospital Universitario Doctor

Peset

Gaspar Aguilar 90 street

46018 Valencia

Spain

Tel.: + 34/667/523904

Fax: +34/961/622 534

ripolles_tom@gva.es

\section{Abstract}

Aim: The aim was to prospectively evaluate the positive predictive value of ultrasound in the diagnosis of ischemic colitis, with colonoscopy as the reference standard.

Methods: During a 2-year period we included consecutive patients over 50 years of age with sudden abdominal pain and/or rectal bleeding who underwent abdominal sonography in an emergency setting with a thickened segment of colon with a length of more than $10 \mathrm{~cm}$. This clinical-sonographic triad was considered diagnostic for ischemic colitis. A thickened bowel location or color Doppler flow findings on ultrasound examination were evaluated but were not considered to make the diagnosis of ischemic colitis. Basic descriptive statistics were used to characterize the study patients. The positive predictive value was calculated as: number of patients with

\section{Introduction \\ $\nabla$}

Ischemic colitis is the most common form of gastrointestinal ischemia. In most cases it consists of non-occlusive ischemia of the bowel caused by a sudden drop in blood flow in the small vessels of the colon, generally secondary to a low-flow state $[1,2]$. From a clinical and histological point of view, it may be classified as 2 major forms: non-gangrenous and gangrenous [1-3]. The nongangrenous form accounts for $80-85 \%$ of cases and is a transient and self-limiting disease in most of patients. The gangrenous form has high morbidity and mortality because it can be associated with transmural necrosis and requires urgent laparotomy [1-3].

Although it can occur at almost any age, approximately $90 \%$ of the cases of colonic ischemia occur in patients over 60 years of age [4-7]. Colonic ischemia is clinically suspected when a patient manifests the classic presentation of abrupt the definitive diagnosis of ischemic colitis (colonoscopic or follow-up)/number of patients with a sonographic diagnosis of ischemic colitis.

Results: A total of 48 patients had the clinicalsonographic triad; mean age: 74.3 years (range 54-90 years). The most frequent clinical manifestation was rectal bleeding ( $83 \%$ of the cases) followed by abdominal pain (81\%) and diarrhea ( $45 \%)$. A total of 42 cases of ischemic colitis were confirmed by endoscopy $(n=35)$ and biopsy $(n=34)$ or clinical evaluation $(n=7)$. The positive predictive value of the clinical-sonographic diagnosis of ischemic colitis was $87.5 \%$. Thickening of the colon wall was detected on the left side in 35 patients with ischemic colitis, on the right side in 3 and pancolitis was identified in 5 patients.

Conclusion: In an appropriate clinical setting, ultrasound has a high positive predictive value for the diagnosis of ischemic colitis.

onset of mild to moderate lower abdominal pain, diarrhea and/or rectal bleeding. However, these 3 symptoms are not specific by themselves and the differential diagnosis from chronic inflammatory bowel disease, infectious colitis, non-steroidal anti-inflammatory drug colitis, diverticulitis, colorectal tumors or radiation colitis should be made [8].

Colonoscopy, although invasive, is the gold standard for final diagnosis $[9,10]$. Biopsy features are nonspecific. Therefore, they must be combined with clinical symptoms and patient history to improve further diagnosis $[5,10]$. In daily practice, it is not usual to perform a colonoscopy in an emergency setting or in elderly patients since the procedure may have complications in cases of severe illness or high-risk patients [11]. Moreover, colonoscopy can be non-diagnostic if it is performed in an emergency setting without previous preparation $[5,11]$. 


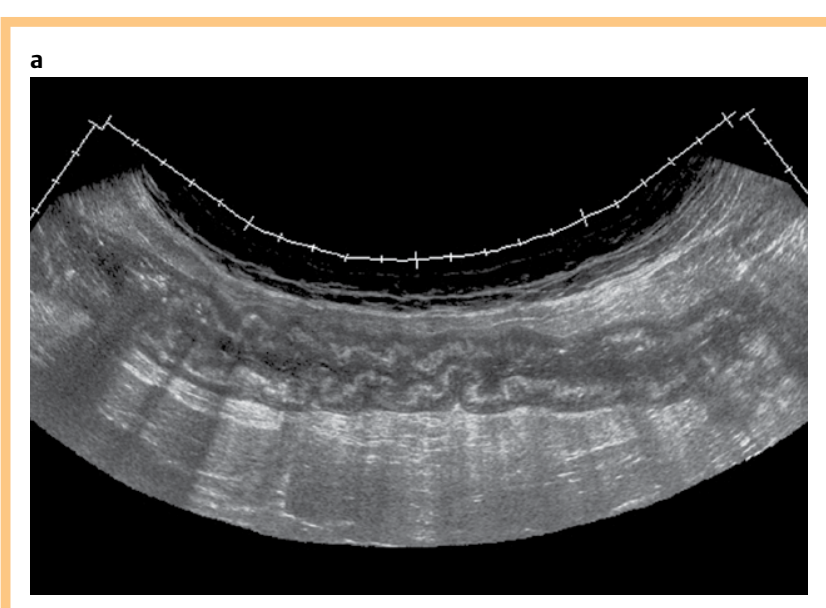

b

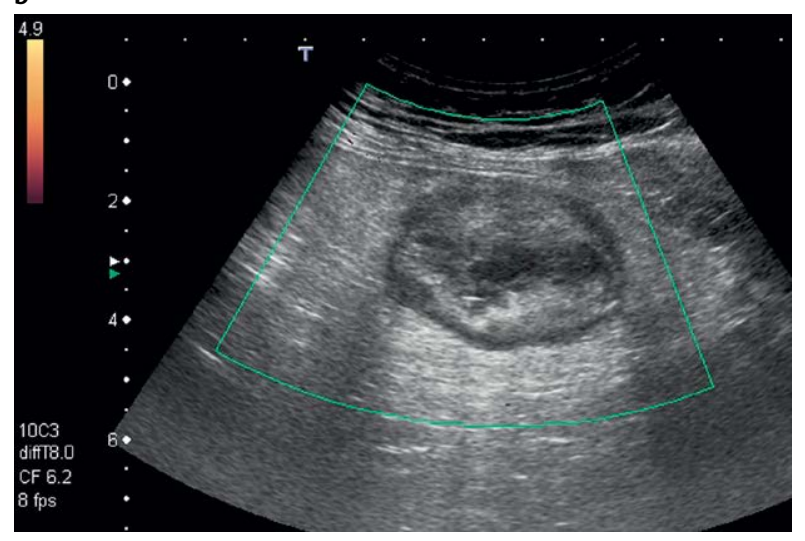

Fig. 1 Ischemic colitis in 74-year-old man with abdominal pain, rectal bleeding and diarrhea. a Compound sonographic image shows thickening of bowel wall of descending and sigmoid colon, with preservation of wall stratification and increased echogenicity of the pericolic fat. b Transverse sonogram shows absence of color Doppler flow. Diagnosis was confirmed by colonoscopy.

Imaging techniques, such as ultrasound and $\mathrm{CT}$, are frequently the first test used in hospitals to evaluate patients with abdominal disorders. US and CT are able to detect abnormalities in the colon wall, so they can play a role in the diagnosis of ischemic colitis. The advantages of these tests are obvious: they are noninvasive and readily available and no preparation is required. CT and ultrasonographic findings have been previously described in the radiological literature [12-18]. However, to our knowledge, the accuracy of these imaging techniques in the diagnosis of ischemic colitis has not been previously assessed. Obviously, the sensitivity and specificity of these imaging techniques in the diagnosis of ischemic colitis (relative to colonoscopy or surgery) cannot be measured in clinical practice. The reason for this is that many negative CT and US cases are not referred to colonoscopy or surgery and thus cannot be stratified into true-negative vs. false-negative results. However, because both true-positive and false-positive imaging results can be followed in clinical practice, the positive predictive value (PPV) of imaging screening can be obtained.

The aim of our study was to prospectively evaluate the positive predictive value (PPV) of abdominal sonography in the diagnosis of ischemic colitis, with colonoscopy as the reference standard.
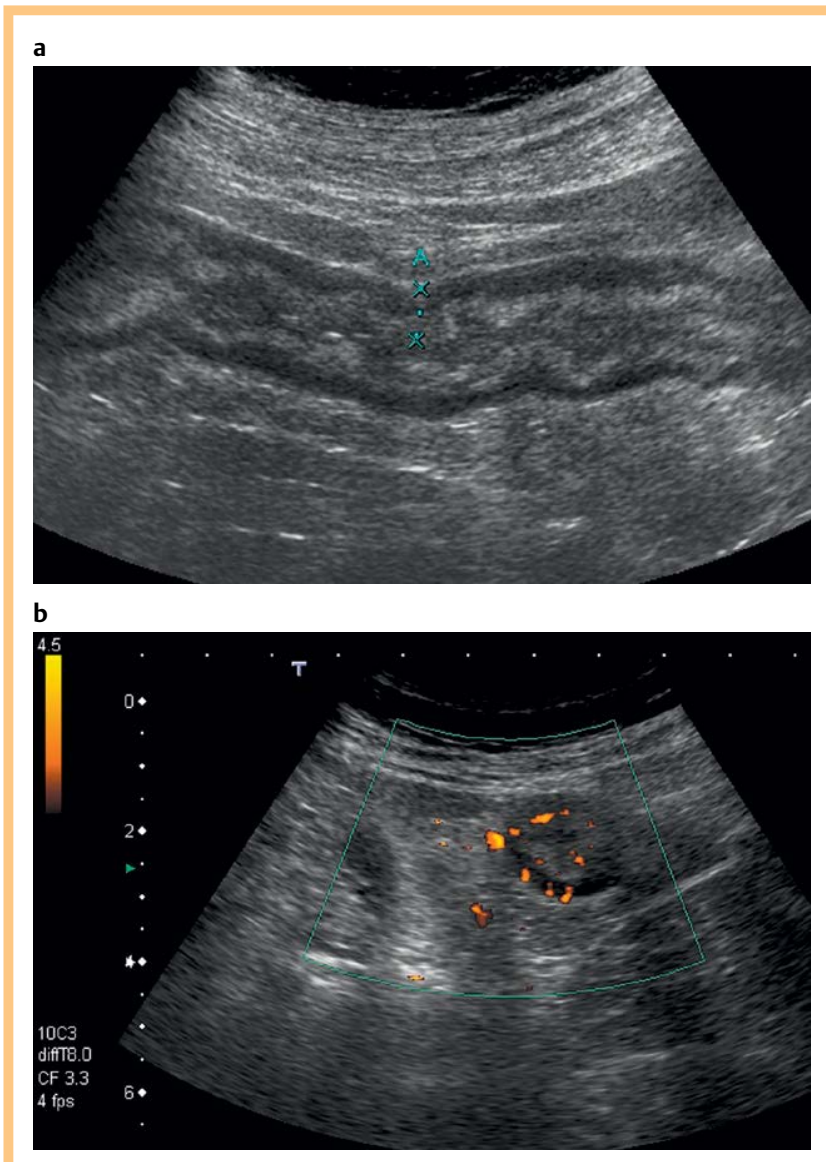

Fig. 2 Ischemic colitis in 77-year-old woman with abdominal pain and rectal bleeding. a Sonogram reveals marked thickening of the left colon with normal stratification. b Transverse sonogram shows moderate vascularity on color Doppler flow. Diagnosis was confirmed by colonoscopy and biopsy.

\section{Materials and Methods}

$\nabla$

This is a prospective study performed during a period of 2 years (between June 2012 and June 2014) that included all consecutive patients over 50 years of age admitted to the gastroenterology department of our hospital with sudden acute abdominal pain and/or rectal bleeding and a sonographic examination with the detection of a thickened ( $>3 \mathrm{~mm}$ ) segment of colon with a length of more than $10 \mathrm{~cm}(\bullet$ Fig. 1,2) $[14,19]$. This triad was considered diagnostic for ischemic colitis, independently of the clinical presentation or initial presumptive diagnosis. Ultrasonographic criteria used for the diagnosis of ischemic colitis were based on previously published data [14]. The location of the thickened bowel or color Doppler flow findings were not considered to make the diagnosis of ischemic colitis. We got oral informed consent from each study participant and the local ethics committee of our hospital approved the study protocol ( $\bullet$ Fig. 3 ).

All studies were conducted with a Toshiba Aplio 80 (Toshiba, Tokyo, Japan), employing a $3.5-5 \mathrm{MHz}$ or a $5-10 \mathrm{MHz}$ convexarray transducer. 3 radiologists with broad experience in bowel ultrasound performed the sonographic examinations. A systematic overview of the colon was performed in each patient, beginning with grayscale US. First the ascending colon and cecum were identified in the right lower quadrant. Then the colon was followed along the transverse colon, descending portion and sig- 
moid colon. When assessing the colon, compression of bowel segments was usually applied in order to displace interfering gas. Bowel wall vascularity by color Doppler US with a special preset optimized for slow flow detection was then evaluated (filter at low setting, [ $50 \mathrm{~Hz}$ ] and lowest velocity scale [2-5 cm/sec], designed for the detection of low-velocity flow in the bowel wall). Color Doppler flow was considered present when color pixels persisted throughout the observation period. Color Doppler flow was always confirmed by obtaining an arterial or venous signal at the location of the color pixel. The intensity of the color Doppler flow was subjectively graded as absent (grade 0 ), barely visible vascularity (grade 1 ), moderate vascularity (grade 2 ) and marked vascularity (grade 3 ).

Grayscale and color Doppler ultrasound were used to assess the following findings: location (left or right side, or pancolitis), length of the affected segment, parietal thickness, stratified or non-stratified bowel wall echotexture, presence of ascites, abnormal echogenicity of pericolic fat and color Doppler grade. The clinical findings (abdominal pain, diarrhea, fever or rectal bleeding) and the sonographic findings of each patient were prospectively recorded by the radiologist filling in a questionnaire at the end of sonography. Patients with fever were excluded from the study.

Colonoscopy was performed as part of the diagnostic work-up and was considered the reference standard. The diagnosis of ischemic colitis was confirmed by the endoscopic findings. If it was not possible to carry out colonoscopy, the definitive diagnosis was made based on typical clinical findings, negative stool culture results, risk factors and clinical and sonographic follow-up examination. Basic descriptive statistics including the mean, standard deviation and maximum and minimum value for continuous variables, and the absolute frequency and percentage for discrete variables were used to characterize the study patients. The PPV was calculated as: number of patients with definitive diagnosis of ischemic colitis (colonoscopy or follow-up)/number of patients with an ultrasound diagnosis of ischemic colitis.

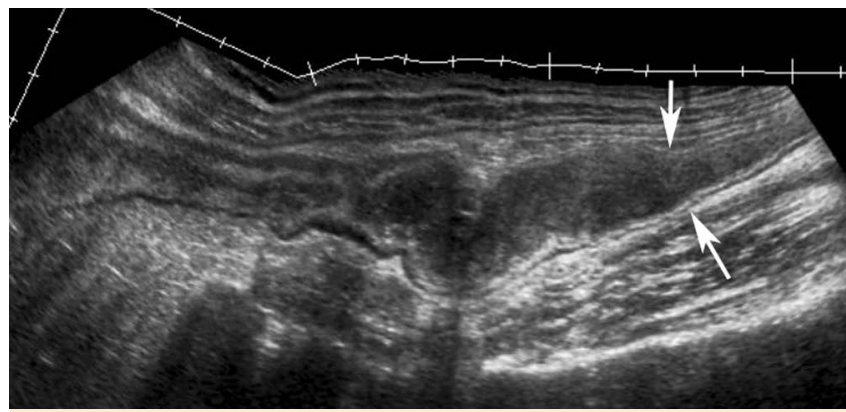

Fig. 3 Ischemic colitis in an 85-year-old man who presented with abdominal pain and diarrhea. Compound sonographic image depicts symmetric thickening of the left colon. The transition from the normal colonic wall to the thickened colonic wall can be seen (arrows).

\section{Results}

$\nabla$

A total of 48 patients had the clinical-sonographic triad and were included in the study group (15 men and 33 women; mean age: 74.3 ; range: $54-90$ years). The most frequent clinical manifestation was rectal bleeding ( $83 \%$ of cases) followed by abdominal pain (81\%) and diarrhea (45\%). The classic clinical triad of pain, diarrhea and rectal bleeding was only recorded in 16 patients (34\%).

Colonoscopy was performed in 40 (83.3\%) out of the 48 patients and biopsy was carried out in 34 patients. Colonoscopy was not performed in 8 cases due to older age, resolution of symptoms or contraindication of the technique ( 3 patients). There was a diagnosis of ischemic colitis in 35 out of 40 colonoscopies ( 34 with biopsy). The other 5 patients had a different diagnosis: nonspecific colitis ( 3 patients), chronic inflammatory bowel disease ( 1 patient) and diverticular disease (1 patient). The diagnosis in the patients without colonoscopic confirmation was: ischemic colitis in 7 patients and non-specific colitis in 1. A total of 42 cases of ischemic colitis were confirmed, with the PPV of ultrasound being $87.5 \%$. The results were similar comparing the PPV between the group of colonoscopy and biopsy (87.5\%) and those without colonoscopic confirmation (87.5\%).

- Table 1 shows the sonographic findings of the study group. Sonographic thickening of the colon wall was detected on the left side in 39 patients (34 with ischemic colitis) (81.3\%) and on the right side in 5 patients ( 3 with ischemic colitis) and pancolitis was identified in 5 patients (all ischemic colitis) (10.4\%) ( $\triangle$ Table 1). Altered pericolic fat was seen in 21 patients $(50 \%)$ and free fluid in 8 (19\%). Color Doppler flow was absent or barely visible in 29 out of 42 patients with ischemic colitis (69\%) and readily visible in 13 patients (31\%).

Analysis of the results according to the affected segment was as follows: left side colitis was detected in 39 patients (PPV 89.7\%), color Doppler flow was absent or barely visible in 27 out of 39 (69.3\%, PPV 88.8\%) and readily visible color Doppler flow was detected in 12 patients (30.7\%, PPV 91.6\%). Pancolitis was seen in 5 patients (PPV 100\%), and 3 of them had absent or barely visible color Doppler flow. Lastly, thickening was located on the right side in 4 patients (PPV 75\%), 3 of them with absent or barely visible color Doppler flow (PPV 100\%).

\section{Discussion}

\section{$\nabla$}

Our results demonstrate that in the appropriate clinical setting, abdominal sonography has a high positive predictive value for diagnosing ischemic colitis, with a PPV of $87.5 \%$ in our study. The diagnosis of ischemic colitis was based on 3 criteria: advanced age of patients ( $>50$ years old), sudden appearance of

\begin{tabular}{|c|c|c|c|c|}
\hline & & $\begin{array}{l}\text { Total } \\
(n=48)\end{array}$ & $\begin{array}{l}\text { Ischemic colitis } \\
(n=42)\end{array}$ & $\begin{array}{l}\text { Other diagnosis } \\
(n=6)\end{array}$ \\
\hline \multirow[t]{3}{*}{ Location } & Left side & 38 & 34 & 4 \\
\hline & Right side & 5 & 3 & 2 \\
\hline & Pancolitis & 5 & 5 & 0 \\
\hline Length & & $28.34 \pm 23.3$ & $30.7 \pm 26.6$ & $23.3 \pm 10.8$ \\
\hline Thickness & & $6.7 \pm 1.6$ & $6.68 \pm 1.6$ & $6.6 \pm 2$ \\
\hline \multirow[t]{2}{*}{ Color Doppler grade } & Absence or barely visible & $32(67 \%)$ & $29(69 \%)$ & $3(50 \%)$ \\
\hline & Readily visible & $16(33 \%)$ & $13(31 \%)$ & $3(50 \%)$ \\
\hline
\end{tabular}

Table 1 Sonographic findings in 48 patients with suspicion of ischemic colitis. 
symptoms and sonographic detection of parietal thickening of a colonic segment with a length of more than $10 \mathrm{~cm}$. It is noteworthy that we have not studied the sensitivity of ultrasound for the diagnosis of ischemic colitis. We are certainly aware that the absence of ultrasound abnormalities does not exclude ischemic colitis (8).

To calculate PPV, we did not assess other features of ischemic colitis described in previous publications [14,17,20,21], such as the location of the affected segment or color Doppler flow grade. Had we included these imaging findings in our study, only the location of the thickened wall would have increased the PPV of sonography, but not significantly. When just the parietal left colon was thickened, the PPV increased up to $89.7 \%$ and in cases of pancolitis it reached $100 \%$. Color Doppler flow findings did not improve the PPV. If we had considered the absence or decrease of the color Doppler flow as a diagnostic criterion of ischemic colitis, the PPV would have been 69\%, i.e., much lower than $87.5 \%$. However, it is surprising that all cases with thickening of the right colon with a barely visible color Doppler flow were confirmed as ischemic colitis (nevertheless, our series is not large enough to validate these results).

Our results agree with those reported for CT in the diagnosis of acute bowel ischemia $[15,16,18]$. We have to take into account that the aim of previous CT studies was to detect intestinal ischemia or infarction in both the small and large bowel, whereas in our study, colonic ischemia was the only location considered. As far as we know, the accuracy or the PPV of CT in the diagnosis of ischemic colitis has not been evaluated yet.

In accordance with other publications $[12,14,15,17]$, a segmental distribution was present in the majority of the patients in our series, with the left colon being the most frequently affected location. The mean length of involvement was $28.34 \mathrm{~cm}$ and the mean parietal thickness was $6.7 \mathrm{~mm}$. Balthazar et al. [12] described a segmental distribution in $89 \%$ of their cases. The left colon was affected in $70 \%$ of their patients; the mean length of involvement was $20 \mathrm{~cm}$; and the mean parietal thickness was $8 \mathrm{~mm}$. Our percentage of patients with absent or barely visible color Doppler flow (69\%) was slightly lower than the one observed by Ripollés et al. [14] and Teefey et al. [21] with 80 and $86 \%$ of patients, respectively.

Ischemic colitis has been described as the most frequent vascular disease in patients over the age of 65 years $[1,14,22]$. However, it is believed to be an underestimated disease in daily clinical practice because of its wide spectrum of clinical symptoms, the non-specificity of clinical and laboratory tests and the transient course of the disease [22-24]. Therefore, ischemic colitis is a clinical challenge that needs complementary examinations to make the correct diagnosis. Ultrasound and CT are widely used to study patients with abdominal pain. Both techniques can detect mural thickening of the colon (the main finding of ischemic colitis), and therefore can suggest this pathology in the appropriate clinical setting. The advantage of CT imaging is its ability to easily detect intestinal pneumatosis, a rare finding in the course of this disease ( $6 \%$ in the series of Balthazar et al.) $[12,14,18,21]$. CT is also better than ultrasound for showing cases of ischemic colitis following aortic surgery, since the bowel wall is often not thickened in these patients and the diagnosis is based on the absence of contrast enhancement of the involved bowel. In contrast, ultrasound does not require preparation or enema, offers better information of the parietal stratification, is more sensitive for showing milder forms of parietal thickening and avoids the use of iodinated contrast media [8].
We believe that ultrasound could be the initial imaging tool in most cases (except for intestinal pneumatosis and secondary cases of aortic surgery). Our results show that we can suspect ischemic colitis when parietal thickening with a length of more than $10 \mathrm{~cm}$ is found in the appropriate clinical setting. It is noteworthy that the sensitivity of ultrasound for detecting parietal thickening in ischemic colitis can be up to $93 \%$ [14].

However, we have to bear in mind that parietal thickening of the colon is a non-specific finding both in ultrasound and CT, since it may be found in inflammatory, neoplastic and infectious diseases. We emphasize that there were no cases of cancer or diverticulitis in our series. Diverticulitis generally involves the sigmoid colon with a marked extrinsic echogenic area around diverticula representing inflamed peridiverticular fat, whereas ischemic colitis is rarely located in the sigmoid colon only. Sonography can differentiate ischemia from tumor because most primary neoplasms of the gastrointestinal tract present as a short $(<10 \mathrm{~cm})$ asymmetric segment of bowel wall thickening with abrupt margins [25]. However, if ischemic colitis is secondary to colonic carcinoma, it may be impossible to distinguish between the neoplastic and ischemic segments. Patients with ischemic colitis and increased vascularization on color Doppler can be indistinguishable from patients with active inflammatory bowel disease as happened in one of our patients. Differentiation between ischemic colitis and inflammatory bowel disease can also be difficult by endoscopy $[10,21]$.

Ischemic colitis, in the absence of transmural necrosis, is a selflimiting disease with a quick recovery of the mucosal lesions, so if colonoscopy is not carried out immediately, results could be normal. Consequently, the finding of a normal bowel wall in the sonographic follow-up examination reinforces the diagnosis of ischemic colitis $[10,14]$.

On the basis of our results and since ultrasound diagnosis has a high PPV and most cases of ischemic colitis are transient, we think that colonoscopy could be avoided in certain select cases, especially in older or very ill patients. Patients with no improvement at serial sonographic examination need further evaluation with other imaging or endoscopic techniques to confirm the diagnosis and exclude neoplasia as a cause of the ischemia.

The main limitation of our study is the small sample size and the lack of a sensitivity and specificity analysis. We do not know how many patients had a negative ultrasound examination and a colonoscopic diagnosis of ischemic colitis (false-negative cases). A study comparing the accuracy of CT and ultrasound would also be useful, since the latter is an innocuous test, it does not require any additional preparation, it does not irradiate and, most importantly, it avoids the nephrotoxicity of iodinated contrast media, which is especially important in older people. In the present study we did not assess the prognostic value of the different ultrasound findings, such as ascites, abnormal echogenicity of the pericolic fat or the presence of color Doppler flow, as we did not continue monitoring patients once they were discharged. The exclusion of patients admitted to the ICU or surgery is a bias, because these patients may have different findings than the group studied. Finally, we did not record the time between the onset of symptoms and the date of colonoscopy. In conclusion, in routine clinical practice many cases of ischemic colitis are considered to have non-specific abdominal pain, so an emergency imaging examination (ultrasound or CT) is usually carried out without a specific clinical suspicion. In our experience ischemic colitis can be suspected with high reliability (high PPV) when an elderly patient with sudden abdominal pain and/ 
or rectal bleeding shows segmental wall thickening of a long colonic segment $(>10 \mathrm{~cm})$ in the sonographic examination. Because endoscopic features of ischemic colitis are nonspecific, this can help to improve further diagnosis. Moreover, our results support that ultrasound could be considered an alternative diagnostic technique to colonoscopy in high-risk cases or patients with contraindications.

\section{References}

1 Brandt LJ, Boley SJ, Goldberg L et al. Colitis in the elderly. Am J Gastroenterol 1981; 76: 239-245

2 Brandt LJ, Feuerstadt P, Blaszka MC. Anatomic patterns, patient characteristics, and clinical outcomes in ischemic colitis: a study of 313 cases supported by histology. Am J Gastroenterol 2010; 5: 2245-2252

3 Baixauli J, Kiran RP, Delaney CP. Investigation and management of ischemic colitis. Cleve Clin J Med 2003; 70: 920-934

4 Binns JC, Isaacson P. Age-related changes in the colonic blood supply. Gut 1978; 19: 384-390

5 Brandt LJ, Boley SJ. Ischemic and vascular lesions of the bowel. In: Sleisenger MH, Fordtran SJ (Eds.). Gastrointestinal disease: pathophysiology, diagnosis, management. Philadelphia, Pa: Saunders; 1993: 1940-1945

6 Guttormson NL, Bubrick MP. Mortality from ischemic colitis. Dis Colon Rectum 1989; 32: 469-472

7 Zimmerman BJ, Granger DN. Reperfusion injury. Surg Clin North Am 1992; 72: 65-83

8 Taourel P, Aufort S, Merigeaud S et al. Imaging of ischemia colitis. Radiol Clin North Am 2008; 46: 909-924

9 Montoro Huguet MA, Santolaria Piedrafita S. Diagnostic approach to ischemic colitis. Gastroenterol Hepatol 2006; 29: 636-646

10 Zou X, Cao J, Yao Y et al. Endoscopic findings and clinicopathologic characteristics of ischemic colitis: a report of 85 cases. Dig Dis Sci 2009; 54: 2009-2015

11 UpToDate. Lee L, Saltzman JR. Overview of colonoscopy in adults [Homepage]. Uptodate Walham; Massachusetts: May 2015

12 Balthazar EJ, Yen BC, Gordon RB. Ischemic Colitis: CT Evaluation of 54 Cases. Radiology 1999; 211: 381-388
13 Ruiz-Guanter A, Ripollés T, Simó L et al. Colitis isquémica: hallazgos en TC. Radiología 2002; 44: 113-117

14 Ripollés T, Simó L, Martínez MJ et al. Sonographic findings in ischemic colitis in 58 patients. AJR 2005; 184: 777-785

15 Romano $S$, Lassandro $F$, Scaglione $M$ et al. Ischemia and infarction of the small bowel and colon: spectrum of imaging findings. Abdom Imaging 2006; 31: 277-292

16 Romano S, Romano L, Grassi Rl. Multidetector row computed tomography findings from ischemia to infarction of the large bowel. Eur J Radiol 2007; 61: 433-441

17 Dietrich CF, Lembcke B, Jenssen C et al. Intestinal Ultrasound in Rare Gastrointestinal Diseases, Update, Part 1. Ultraschall in Med 2014; 35: 400-421

18 Wiesner $W$, Hauser A, Steinbrich W. Accuracy of multidetector row computed tomography for the diagnosis of acute bowel ischemia in a non-selected study population. Eur Radiol 2004; 14: 2347-2356

19 Nylund $\mathrm{K}$, Hausken T, Gilja $\mathrm{OH}$. Ultrasound and inflammatory bowel disease. Ultrasound Quarterly 2010; 26: 3-15

20 Danse EM, Van Beers BE, Jamart J et al. Prognosis of ischemic colitis: comparison of color Doppler sonography with early clinical and laboratory findings. AJR 2000; 175: 1151-1154

21 Teefey SA, Roarke MC, BrinkJA et al. Bowel wall thickening: differentiation of inflammation from ischemia with color Doppler and duplex US. Radiology 1996; 198: 547-551

22 Brandt LJ, Boley SJ. AGA technical review on intestinal ischemia. Gastroenterology 2000; 118: 954-998

23 Brandt LJ, Boley S. Intestinal Ischemia. In: Feldman M, Friedman L, Sleisenger XX (eds.). Sleisenger-Fordtran Gastrointestinal and Liver Disease. Pathophysiology, Diagnosis and Management. Philadelphia, Pa: Saunders 2002; 2321-2340

24 Sherid M, Sifuentes H, Samo S et al. Ischemic colitis: A forgotten entity. Results of a retrospective study in 118 patients. Journal of digestive diseases 2014; 15: 606-613

25 Ripollés T, Martínez-Pérez MJ, Gómez Valencia DP et al. Sigmoid stenosis caused by diverticulitis vs. carcinoma: usefulness of sonographic features for their differentiation in the emergency setting. Abdom Imaging 2015 May 12. [Epub ahead of print] 